\author{
MITSUBISHI ELECTRIC RESEARCH LABORATORIES \\ http://www.merl.com
}

\title{
Reduced-Rate OFDM Transmission with Statistics-based ICI Mitigation
}

\author{
Ma, J.; Orlik, P.; Zhang, J.; Li, G. Y.
}

TR2011-029 June 2011

\begin{abstract}
In this paper, we develop a general reduced-rate orthogonal frequency division multiplexing (OFDM) transmission scheme for inter-sub-channel interference (ICI) mitigation in a high-mobility environment. By transmit and receive preprocessing, we transform the original OFDM system into an equivalent one with fewer sub-carriers and significantly reduced ICI. A general structure of transmit and receive preprocessing matrices is developed so that all sub-channels in the transformed OFDM system have a common average signal-to-interference ratio (SIR). By utilizing channel statistics, we further optimize the preprocessing coefficients to maximize the SIR. Numerical and simulation results demonstrate that the proposed reduced-rate OFDM transmission achieves significant performance improvement over the existing ICI self-cancellation schemes.
\end{abstract}

IEEE International Conference on Communications (ICC)

This work may not be copied or reproduced in whole or in part for any commercial purpose. Permission to copy in whole or in part without payment of fee is granted for nonprofit educational and research purposes provided that all such whole or partial copies include the following: a notice that such copying is by permission of Mitsubishi Electric Research Laboratories, Inc.; an acknowledgment of the authors and individual contributions to the work; and all applicable portions of the copyright notice. Copying, reproduction, or republishing for any other purpose shall require a license with payment of fee to Mitsubishi Electric Research Laboratories, Inc. All rights reserved. 



\title{
Reduced-Rate OFDM Transmission with Statistics-based ICI Mitigation
}

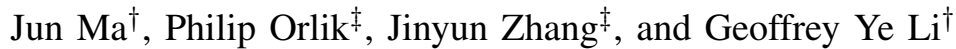 \\ ${ }^{\dagger}$ School of Electrical and Computer Engineering, Georgia Tech, Atlanta, GA 30332, USA \\ ${ }^{\ddagger}$ Mitsubishi Electric Research Laboratories, Cambridge, MA 02139, USA \\ Email: jma6@mail.gatech.edu, \{porlik,jzhang\}@merl.com, liye@ece.gatech.edu
}

\begin{abstract}
In this paper, we develop a general reduced-rate orthogonal frequency division multiplexing (OFDM) transmission scheme for inter-subchannel interference (ICI) mitigation in a high-mobility environment. By transmit and receive preprocessing, we transform the original OFDM system into an equivalent one with fewer subcarriers and significantly reduced ICI. A general structure of transmit and receive preprocessing matrices is developed so that all subchannels in the transformed OFDM system have a common average signal-to-interference ratio (SIR). By utilizing channel statistics, we further optimize the preprocessing coefficients to maximize the SIR. Numerical and simulation results demonstrate that the proposed reduced-rate OFDM transmission achieves significant performance improvement over the existing ICI self-cancellation schemes.
\end{abstract}

Index Terms-Inter-subchannel interference, OFDM, ratereduction factor, time-varying channel

\section{INTRODUCTION}

Orthogonal frequency division multiplexing (OFDM) modulation avoids intersymbol interference (ISI) by dividing a broadband channel into a number of orthogonal narrowband subchannels. However, variation of the wireless channel within an OFDM symbol destroys the orthogonality and causes intersubchannel interference (ICI) [1], [2], which, if not cancelled, will degrade system performance and result in an error floor.

In a high-mobility environment, the impulse response of channel varies with time even within an OFDM symbol. As a result, the frequency-domain channel matrix is no longer diagonal and the off-diagonal elements are the interference gains among OFDM subchannels. It has been suggested in [3]-[5] to estimate these interference gains and perform ICI cancellation accordingly, which usually requires a large amount of pilots, especially in a high-mobility environment. As an alternative to pilot-assisted ICI cancellation, various OFDM transmission schemes with inherent ICI mitigation capabilities have been proposed. In [6], second-order frequency-domain correlative coding has been proposed to mitigate ICI by introducing correlation among transmitted signals over neighboring OFDM subchannels. In [7], the optimal partial response encoding at an OFDM transmitter that minimizes the ICI power has been proposed as an extension of the frequency-domain correlative coding. However, these pilot-free full-rate OFDM transmission schemes only have limited ICI mitigation capabilities and still suffer considerable residual ICI. To deal with this problem,

This work was supported by a research gift from Mitsubishi Electric Research Laboratories (MERL). an ICI self-cancellation transmission scheme has been developed in [8], which significantly reduces ICI at the expense of a halved spectral efficiency. In [9], a general ICI selfcancellation scheme has been further developed to achieve tradeoffs between ICI mitigation and spectral efficiency.

In this paper, we extend the existing work in [8] and [9] and establish a general reduced-rate OFDM transmission framework with statistics-based ICI self-cancellation. Via transmit and receive preprocessing, we transform the original OFDM system into an equivalent one with fewer subcarriers and significantly reduced ICI level. Without requiring the instantaneous channel state information, we develop a general structure of transmit and receive preprocessing matrices to ensure that all subchannels in the equivalent OFDM system share a common average signal-to-interference ratio (SIR). For the developed structure, we further optimize the transmit and the receive preprocessing coefficients based on channel statistics so as to maximize this common SIR. Numerical and simulation results demonstrate that the proposed reducedrate OFDM transmission achieves significant performance improvement over the existing ICI self-cancellation schemes.

The remainder of this paper is organized as follows. In Section II, we describe the channel model and ICI in highmobility OFDM. Then we present the principle of reduced-rate OFDM transmission in Section III and design the transmit and the receive preprocessing matrices in Section IV, respectively. Numerical and simulation results are presented in Section V. Finally Section VI concludes this paper.

\section{ICI IN HIGH-MOBILITY OFDM}

Consider an $N$-subcarrier OFDM system with sampling interval $T_{s}$. Assume that the maximum delay of the multipath channel is $(L-1) T_{s}$. Without loss of generality, we model the discrete-time multipath channel as an $L$-tap one. Denote $h_{l, n}$ as the channel gain over the $l$ th tap at sampling instant $n$. In a high-mobility environment, $h_{l, n}$ varies with $n$ randomly with the time-domain correlation functions given by

$$
r_{l}(m)=E\left\{h_{l, n+m} h_{l, n}^{*}\right\}=\xi_{l} \phi\left(m T_{s}\right), \quad 0 \leq l \leq L-1,
$$

where $E\{\cdot\}$ denotes the expectation operator, $\xi_{l}$ denotes the average power over the $l$ th tap, and $\phi(\cdot)$ with $\phi(0)=1$ denotes the normalized correlation function of the time-varying channel. Furthermore, we assume that channels over different taps are uncorrelated and normalize the average power gain of the multipath channel so that $\sum_{l=0}^{L-1} \xi_{l}=1$. 


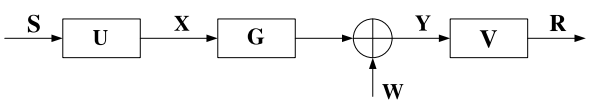

(a) Frequency-domain

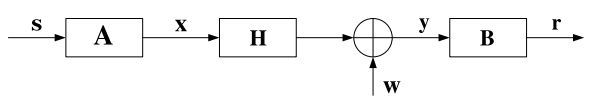

(b) Time-domain

Fig. 1. Block diagram of the reduced-rate OFDM transmission

Denote $\mathbf{X}=\left(X_{0}, X_{1}, \cdots, X_{N-1}\right)^{T}$ as the frequencydomain transmitted signal vector and $\mathbf{F}_{N}$ as the $N \times N$ discrete fourier transform (DFT) matrix with $\mathbf{F}_{N}\left(n_{1}, n_{2}\right)=$ $\frac{1}{\sqrt{N}} e^{-\frac{2 \pi n_{1} n_{2}}{N}}, 0 \leq n_{1}, n_{2} \leq N-1$, and then the time-domain transmitted signal vector is given by $\mathbf{x}=\mathbf{F}_{N}^{H} \mathbf{X}$. Suppose that ISI is avoided by cyclic prefix, and then the time-domain received signal vector is given by $\mathbf{y}=\mathbf{H x}+\mathbf{w}$, where $\mathbf{w}$ denotes the additive white Gaussian noise vector and $\mathbf{H}$ is the $N \times N$ time-domain channel matrix. According to the channel model described above, $\mathbf{H}\left(n_{1}, n_{2}\right)=h_{\left(n_{1}-n_{2}\right)_{N}, n_{1}}$, where $(\cdot)_{N}$ denotes the operation modulo $N$. The corresponding frequency-domain received signal vector can be obtained as

$$
\mathbf{Y}=\mathbf{F}_{N} \mathbf{y}=\mathbf{G X}+\mathbf{W},
$$

where $\mathbf{W}=\mathbf{F}_{N} \mathbf{w}$ denotes the frequency-domain white noise vector and $\mathbf{G}=\mathbf{F}_{N} \mathbf{H} \mathbf{F}_{N}^{H}$ denotes the frequency-domain channel matrix over the $N$ OFDM subchannels. The $(m, k)$ element of $\mathbf{G}$ denotes the channel or interference gain from the $k$ th to the $m$ th OFDM subchannel and can be obtained as

$$
\mathbf{G}(m, k)=\sum_{l=0}^{L-1} \frac{e^{-j \frac{2 \pi k l}{N}}}{N} \sum_{n=0}^{N-1} h_{l, n} e^{-j \frac{2 \pi(m-k) n}{N}},
$$

which indicates that when $m \neq k, \mathbf{G}(m, k)$ is generally not zero unless $h_{l, n}=h_{l}, 0 \leq n \leq N-1$, i.e., variation of the wireless channel within an OFDM symbol causes ICI.

\section{PRINCIPLE OF REDUCED-RATE OFDM TRANSMISSION}

\section{A. Frequency-Domain}

In reduced-rate OFDM transmission, $K$ data symbols are loaded over $N$ subcarriers with $\eta=\frac{N}{K}$ defined as the ratereduction factor (RRF). For a tradeoff between ICI mitigation and spectral efficiency, we assume that $1 \leq \eta \leq 2$. Even so, the developed schematic framework and the presented analysis in this paper can be easily extended to the case of $\eta>2$.

Figure 1(a) shows the frequency-domain block diagram of reduced-rate OFDM transmission, in which $\mathbf{S}$ is the transmitted signal vector over $K$ equivalent subchannels, $\mathbf{U}$ is the $N \times K$ transmit preprocessing matrix, and the actually transmitted signal vector over $N$ OFDM subcarriers is $\mathbf{X}=\mathbf{U S}$. At the receiver, we further perform receive preprocessing to get the received signal vector over $K$ equivalent subchannels as $\mathbf{R}=\mathbf{V Y}$ where $\mathbf{V}$ denotes the $K \times N$ receive preprocessing matrix and $\mathbf{Y}$ is the received signal vector over $N$ OFDM subcarriers given in (2). Thus we obtain the frequency-domain system equation over $K$ equivalent subchannels as

$$
\mathbf{R}=\widetilde{\mathbf{G}} \mathbf{S}+\widetilde{\mathbf{W}}
$$

where $\widetilde{\mathbf{G}}=$ VGU denotes the equivalent channel matrix and $\widetilde{\mathbf{W}}=\mathbf{V W}$ is the received noise vector. Equation (4) indicates that, by reducing the transmission rate via transmit and receive preprocessing, we transform the original $N$-subcarrier OFDM system into an equivalent $K$-subcarrier one.

\section{B. Time-Domain}

Denote $\mathbf{F}_{K}$ as the $K \times K$ DFT matrix, $\mathbf{s}$ and $\mathbf{r}$ as the timedomain transmitted and received signal vectors, respectively, and then the time-domain system equation of the equivalent $K$-subcarrier OFDM system can be obtained from (4) as

$$
\mathbf{r}=\widetilde{\mathbf{H}} \mathbf{s}+\widetilde{\mathbf{w}},
$$

where $\widetilde{\mathbf{H}}=\mathbf{F}_{K}^{H} \widetilde{\mathbf{G}} \mathbf{F}_{K}$ is the equivalent time-domain channel matrix and $\widetilde{\mathbf{w}}=\mathbf{F}_{K}^{H} \widetilde{\mathbf{W}}$. Since $\widetilde{\mathbf{H}}=\mathbf{F}_{K}^{H} \widetilde{\mathbf{G}} \mathbf{F}_{K}, \widetilde{\mathbf{G}}=\mathbf{V G U}$, and $\mathbf{G}=\mathbf{F}_{N} \mathbf{H F}_{N}^{H}$, the mapping from the original to the equivalent time-domain channel matrices can be expressed as

$$
\widetilde{\mathbf{H}}=\mathbf{B H A} \text {, }
$$

where $\mathbf{A}=\mathbf{F}_{N}^{H} \mathbf{U} \mathbf{F}_{K}$ denotes the $N \times K$ time-domain transmit preprocessing matrix and $\mathbf{B}=\mathbf{F}_{K}^{H} \mathbf{V} \mathbf{F}_{N}$ is the $K \times N$ timedomain receive preprocessing matrix. Denote $\tilde{h}_{l, n}, 0 \leq l, n \leq$ $K-1$, as the channel gain over the $l$ th tap at the $n$th sampling instant of the equivalent $K$-subcarrier OFDM system, and then

$$
\widetilde{\mathbf{H}}\left(n_{1}, n_{2}\right)=\tilde{h}_{\left(n_{1}-n_{2}\right)_{K}, n_{1}}, \quad 0 \leq n_{1}, n_{2} \leq K-1 .
$$

Based on (5) and (6), we show the time-domain block diagram of reduced-rate OFDM transmission in Fig. 1(b).

\section{Design of Preprocessing Matrices}

In this section, we will design the transmit preprocessing matrix, $\mathbf{U}$ or $\mathbf{A}$, and the receive preprocessing matrix, $\mathbf{V}$ or $\mathbf{B}$, so that the equivalent $K$-subcarrier OFDM system has significantly reduced ICI.

\section{A. Common SIR over Equivalent Subchannels}

According to (4), the frequency-domain received signal over the $m$ th $(0 \leq m \leq K-1)$ equivalent subchannel is given by

$$
R_{m}=\widetilde{\mathbf{G}}(m, m) S_{m}+\sum_{k=0, k \neq m}^{K-1} \widetilde{\mathbf{G}}(m, k) S_{k}+\widetilde{W}_{m},
$$

where the first and second terms denote the desired signal and the ICI, respectively. Assume that the transmitted signals over different equivalent subchannels, $S_{k}$ 's, are independent with zero mean and variance $\sigma_{s}^{2}$, and then the average SIR over the $m$ th equivalent subchannel can be obtained as

$$
\operatorname{SIR}_{m}=\frac{E\left\{|\widetilde{\mathbf{G}}(m, m)|^{2}\right\}}{\sum_{k=0, k \neq m}^{K-1} E\left\{|\widetilde{\mathbf{G}}(m, k)|^{2}\right\}}=\frac{P(m, m)}{\sum_{k=0, k \neq m}^{K-1} P(m, k)},
$$

where $P(m, k)$ denotes the average power gain from the $k$ th to the $m$ th equivalent subchannel. Because of the circular and symmetric structure of the OFDM modulation, it is expected that, with the optimal preprocessing matrices, the average power gain from the $k$ th to the $m$ th equivalent subchannel 
only depends on the distance between the two subchannels, i.e., $P(m, k)=p\left((m-k)_{K}\right)$, where $p(\cdot)$ denotes a onedimensional function, and all of the $K$ equivalent subchannels have the same SIR given by

$$
\mathrm{SIR}=\frac{p(0)}{\sum_{n=1}^{K-1} p(n)} .
$$

Since $\widetilde{\mathbf{G}}=\mathbf{F}_{K} \widetilde{\mathbf{H}} \mathbf{F}_{K}^{H}$ and $\widetilde{\mathbf{H}}\left(n_{1}, n_{2}\right)=\tilde{h}_{\left(n_{1}-n_{2}\right)_{K}, n_{1}}$,

$$
\begin{aligned}
P(m, k)= & \frac{1}{K^{2}} \sum_{l_{1}=0}^{M-1} \sum_{l_{2}=0}^{M-1} e^{-j \frac{2 \pi k}{K}\left(l_{1}-l_{2}\right)} \\
& \cdot \sum_{n_{1}=0}^{K-1} \sum_{n_{2}=0}^{K-1} E\left\{\tilde{h}_{l_{1}, n_{1}} \tilde{h}_{l_{2}, n_{2}}^{*}\right\} e^{-j \frac{2 \pi(m-k)\left(n_{1}-n_{2}\right)}{K}},
\end{aligned}
$$

which indicates that $P(m, k)$ generally depends on both $m$ and $k$. However, if $E\left\{\tilde{h}_{l_{1}, n_{1}} \tilde{h}_{l_{2}, n_{2}}^{*}\right\}=0$ for any $l_{1} \neq l_{2}$, it can be shown that $P(m, k)$ will only depend on $(m-k)_{K}$. Thus we obtain the following proposition.

Proposition 1: The equivalent subchannels have a common SIR if different taps in the time-domain channel of the transformed $K$-subcarrier OFDM system are uncorrelated.

\section{B. Structure of $\mathbf{A}$ and $\mathbf{B}$ for a Common SIR}

According to Proposition 1, we need to design the timedomain preprocessing matrices, $\mathbf{A}$ and $\mathbf{B}$, so that different taps in the transformed time-domain channel, $\widetilde{\mathbf{H}}=\mathbf{B H A}$, are uncorrelated. From $\widetilde{\mathbf{H}}=\mathbf{B H A}$, it can be obtained that

$$
\widetilde{\mathbf{H}}(i, j)=\sum_{n_{1}=0}^{N-1} \sum_{n_{2}=0}^{N-1} \mathbf{B}\left(i, n_{1}\right) \mathbf{H}\left(n_{1}, n_{2}\right) \mathbf{A}\left(n_{2}, j\right),
$$

for any $0 \leq i, j \leq K-1$. Define $i=(j+l)_{K}$ and $n_{1}=$ $\left(n_{2}+\Delta\right)_{N}$ where $l$ and $\Delta$ denote the tap indexes in $\widetilde{\mathbf{H}}$ and $\mathbf{H}$, respectively, and then (12) can be rewritten as

$$
\begin{aligned}
\widetilde{\mathbf{H}}\left((j+l)_{K}, j\right)= & \sum_{\Delta=0}^{L-1} \sum_{n_{2}=0}^{N-1} \mathbf{B}\left((j+l)_{K},\left(n_{2}+\Delta\right)_{N}\right) \\
& \cdot \mathbf{H}\left(\left(n_{2}+\Delta\right)_{N}, n_{2}\right) \mathbf{A}\left(n_{2}, j\right),
\end{aligned}
$$

where $L$ denotes the number of taps in $\mathbf{H}$, i.e., $\mathbf{H}\left(\left(n_{2}+\Delta\right)_{N}, n_{2}\right)=0$ for any $n_{2}$ if $\Delta \geq L$. Since $L$ is usually much smaller than $N$, we assume it is also smaller than $K$. Equation (13) indicates how tap $\Delta$ in $\mathbf{H}$ is mapped to tap $l$ in $\widetilde{\mathbf{H}}$ via transmit and receive preprocessing. Since different taps in the original time-domain channel, $\mathbf{H}$, are uncorrelated, taps in the transformed time-domain channel, $\widetilde{\mathbf{H}}$, will be still uncorrelated if any tap in $\mathbf{H}$ is mapped to at most one of the taps in $\widetilde{\mathbf{H}}$. Thus we obtain the following proposition.

Proposition 2: The equivalent subchannels have a common SIR if any tap in the original time-domain channel is mapped to at most one of the taps in the transformed one.

Equation (13) indicates that, to guarantee that the channel sequence over tap $\Delta$ in $\mathbf{H}, \mathbf{H}\left(\left(n_{2}+\Delta\right)_{N}, n_{2}\right), 0 \leq n_{2} \leq$ $N-1$, is mapped to only one channel sequence in $\widetilde{\mathbf{H}}$, we need to ensure that each row of $\mathbf{A}$ and each column of $\mathbf{B}$ have at most one non-zero element. Without loss of generality, we let

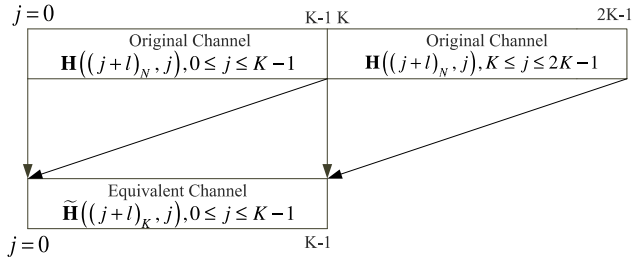

(a) $K=\frac{N}{2}$

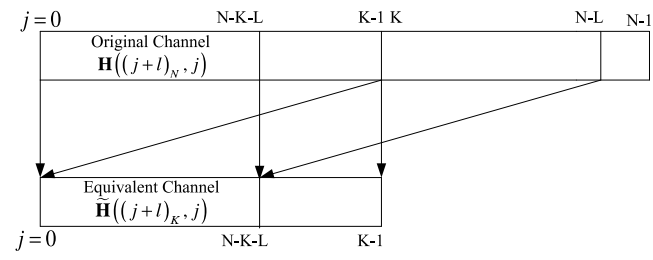

(b) $\frac{N}{2}<K<N$

Fig. 2. Channel mapping over tap $l$.

the first tap in $\mathbf{H}$ be mapped to only the first tap in $\widetilde{\mathbf{H}}$ and construct $\mathbf{A}$ as

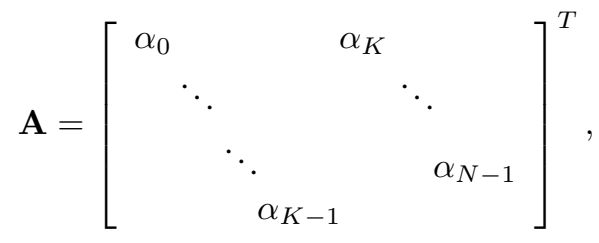

and $\mathbf{B}$ as

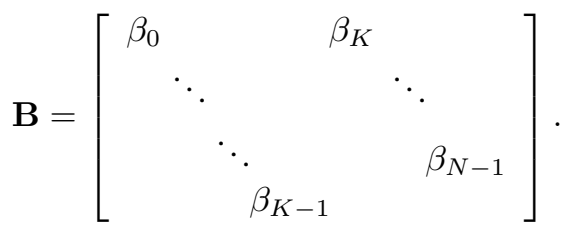

Given the tentative structure of A and $\mathbf{B}$ in (14) and (15), respectively, the following proposition can be proved [10].

Proposition 3: When $K=\frac{N}{2}$, there exists a one-to-one tap mapping from $\mathbf{H}$ to $\widetilde{\mathbf{H}}$ expressed in (16) and a common SIR over $K$ equivalent subchannels for arbitrary $\alpha_{0}, \alpha_{1}, \cdots, \alpha_{N-1}$ and $\beta_{0}, \beta_{1}, \cdots, \beta_{N-1}$.

Based on (16), we show the channel mapping when $K=\frac{N}{2}$ in Fig. 2(a). As indicated, the equivalent channel sequence is obtained by dividing the original one into two segments with equal length and then performing a weighted summation of the two segments. Since the original channel over each tap usually varies with time linearly within an OFDM symbol, it is possible to design these weights appropriately so that the equivalent channel has the least time-domain variation. Therefore, with $K=\frac{N}{2}$, the reduced-rate OFDM transmission is expected to mitigate ICI dramatically.

To guarantee a common SIR over all equivalent subchannels in the case of a fractional RRF, we design $K$ so that $L \leq N-$ $K+1$ and let $\alpha_{n}=0$ for any $N-(L-1) \leq n \leq N-1$. Then, given the structure of A and B in (14) and (15), respectively, the following proposition can be proved [10].

Proposition 4: When $\frac{N}{2}<K<N$, there exists a one-to-one tap mapping from $\mathbf{H}$ to $\widetilde{\mathbf{H}}$ expressed in (17), where $\mathrm{I}_{j \leq N-K-L}$ denotes the indicator function, and a common SIR over $K$ equivalent subchannels for arbitrary 


$$
\begin{aligned}
& \widetilde{\mathbf{H}}\left((j+l)_{K}, j\right)=\alpha_{j} \beta_{j+l} \mathbf{H}(j+l, j)+\alpha_{j+K} \beta_{(j+K+l)_{N}} \mathbf{H}\left((j+K+l)_{N}, j+K\right), \quad 0 \leq j, l \leq K-1 . \\
& \widetilde{\mathbf{H}}\left((j+l)_{K}, j\right)=\alpha_{j} \beta_{j+l} \mathbf{H}(j+l, j)+\mathrm{I}_{j \leq N-K-L} \alpha_{j+K} \beta_{j+K+l} \mathbf{H}(j+K+l, j+K), 0 \leq j, l \leq K-1 .
\end{aligned}
$$

$\alpha_{0}, \alpha_{1}, \cdots, \alpha_{N-L}$ and $\beta_{0}, \beta_{1}, \cdots, \beta_{N-1}$.

Based on (17), we show the channel mapping when $\frac{N}{2}<$ $K<N$ in Fig. 2(b). As indicated, only the first part of the equivalent channel sequence is a weighted summation of two original channel segments while the second part is not. As a result, even if the original channel over each tap varies with time linearly, the variation of the equivalent channel in the second part cannot be eliminated entirely. Therefore, with $K>$ $\frac{N}{2}$, the reduced-rate OFDM transmission increases the spectral efficiency at the expense of degraded ICI mitigation capability.

\section{Optimization of $\mathbf{A}$ and $\mathbf{B}$}

For the developed structure of $\mathbf{A}$ and $\mathbf{B}$, the common average SIR over equivalent subchannels is given by

$$
\mathrm{SIR}=\frac{P(m, m)}{\sum_{k=0, k \neq m}^{K-1} P(m, k)}, \quad 0 \leq m \leq K-1 .
$$

By utilizing the channel statistics, we further optimize the preprocessing coefficient vectors, $\boldsymbol{\alpha}$ and $\boldsymbol{\beta}$, to maximize the SIR over equivalent subchannels. Without loss of generality, we consider the first equivalent subchannel over which the average desired signal and ICI powers $\left(P_{d}\right.$ and $\left.P_{I}\right)$ are given by (19) and (20), respectively, where $f\left(r_{1}, c_{1}, r_{2}, c_{2}\right)=$ $E\left\{\mathbf{G}\left(r_{1}, c_{1}\right) \mathbf{G}^{*}\left(r_{2}, c_{2}\right)\right\}$, which, based on the channel statistics described in Section II, can be obtained as in (21). Thus the optimization problem can be formulated as

$$
\begin{array}{cl}
\max _{\boldsymbol{\alpha}, \boldsymbol{\beta}} & \operatorname{SIR}=\frac{P_{d}}{P_{I}} \\
\text { s.t. } & \sum_{n=0}^{N-1}\left|\alpha_{n}\right|^{2}=N \text { and } \sum_{n=0}^{N-1}\left|\beta_{n}\right|^{2}=N
\end{array}
$$

where the first constraint on $\boldsymbol{\alpha}$ guarantees a constant total transmit power over $N$ OFDM subcarriers and the second constraint on $\boldsymbol{\beta}$ ensures that all $K$ equivalent subchannels share a constant received noise power. Since the above optimization problem is rather complicated, a closed-form solution cannot be obtained analytically. Therefore, we apply the gradientbased steepest descent algorithm to search for the optimal $\boldsymbol{\alpha}$ and $\boldsymbol{\beta}$ numerically. Since the optimization of $\mathbf{A}$ and $\mathbf{B}$ is based on long-term channel statistics, it can be conducted offline, thus relieving the burden of computation complexity.

\section{Numerical AND Simulation Results}

In this section, we present numerical and simulation results to demonstrate the performance of reduced-rate OFDM transmission. A 32-subcarrier $(N=32)$ OFDM system is considered. We let $L=4$ and assume that the average power gains over the 4 taps decay with delay exponentially with the exponent factor $\lambda=0.5$. Furthermore, we assume the classical Jakes' Doppler spectrum over all of the taps with the normalized Doppler frequency defined as $F_{d}=\frac{f_{d}}{f_{s}}$ where $f_{d}$ is the absolute Doppler frequency and $f_{s}$ is the subcarrier spacing. The performance of the proposed reduced-rate OFDM

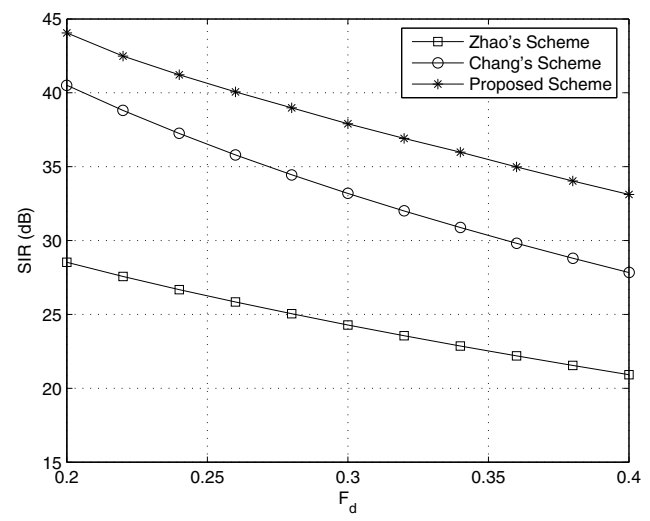

(a) $K=\frac{N}{2}$

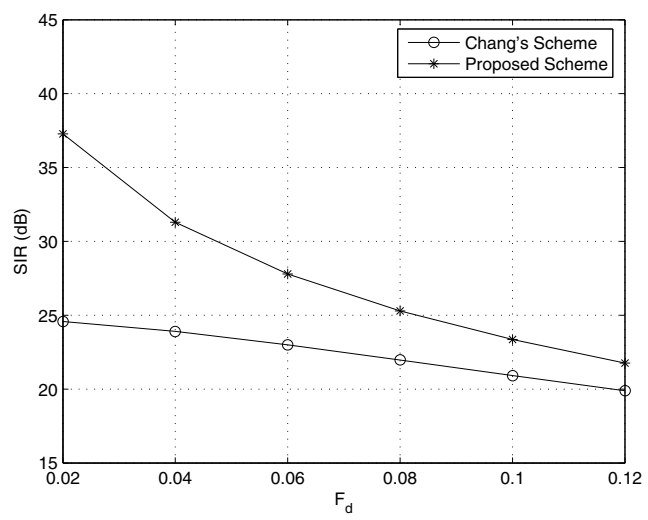

(b) $K=\frac{3}{4} N$

Fig. 3. SIR versus the normalized Doppler frequency $\left(F_{d}\right)$

transmission is demonstrated in comparison with the ICI selfcancellation scheme proposed in [8] for integer RRFs, denoted as the Zhao's scheme, and the improved one proposed in [9] for general RRFs, denoted as the Chang's scheme.

Figure 3(a) shows the SIR versus $F_{d}$ curves of the proposed, the Zhao's, and the Chang's schemes when $K=\frac{N}{2}$. Such a half-rate transmission scheme is suitable for high Doppler frequencies to trade transmission rate for ICI mitigation in a high-mobility environment. It can be shown that both the Zhao's and the Chang's schemes have a common SIR over all equivalent subchannels in the special case of half-rate transmission [10]. As shown in Fig. 3(a), the proposed reduced-rate transmission achieves significant SIR gains of around $15 \mathrm{~dB}$ over the Zhao's scheme and around $5 \mathrm{~dB}$ over the Chang's scheme, respectively. Moreover, we observe that the SIR gain over the Chang's scheme increases with $F_{d}$ slowly.

Figure 3(b) shows the SIR versus $F_{d}$ curves of the proposed and the Chang's schemes when $K=\frac{3}{4} N$. In contrast with the half-rate scheme suitable for high Doppler frequencies, such a $\frac{3}{4}$-rate one is suitable for low Doppler frequencies to achieve a tradeoff between ICI mitigation and spectral efficiency. Since the Chang's scheme does not guarantee a common SIR for 


$$
\begin{gathered}
P_{d}=P(0,0)=\sum_{r_{1}=0}^{N-1} \sum_{c_{1}=0}^{N-1} \sum_{r_{2}=0}^{N-1} \sum_{c_{2}=0}^{N-1} \mathbf{V}\left(0, r_{1}\right) \mathbf{U}\left(c_{1}, 0\right) f\left(r_{1}, c_{1}, r_{2}, c_{2}\right) \mathbf{V}^{*}\left(0, r_{2}\right) \mathbf{U}^{*}\left(c_{2}, 0\right) \\
P_{I}=\sum_{k=1}^{K-1} P(0, k)=\sum_{k=1}^{K-1} \sum_{r_{1}=0}^{N-1} \sum_{c_{1}=0}^{N-1} \sum_{r_{2}=0}^{N-1} \sum_{c_{2}=0}^{N-1} \mathbf{V}\left(0, r_{1}\right) \mathbf{U}\left(c_{1}, k\right) f\left(r_{1}, c_{1}, r_{2}, c_{2}\right) \mathbf{V}^{*}\left(0, r_{2}\right) \mathbf{U}^{*}\left(c_{2}, k\right) \\
f\left(r_{1}, c_{1}, r_{2}, c_{2}\right)=\frac{\sum_{l=0}^{L-1} \xi_{l} e^{j \frac{2 \pi\left(c_{2}-c_{1}\right) l}{N}}}{N^{2}} \sum_{n_{1}=0}^{N-1} \sum_{n_{2}=0}^{N-1} \phi\left(n_{1}-n_{2}\right) e^{j \frac{2 \pi}{N}\left[\left(r_{2}-c_{2}\right) n_{2}-\left(r_{1}-c_{1}\right) n_{1}\right]}
\end{gathered}
$$

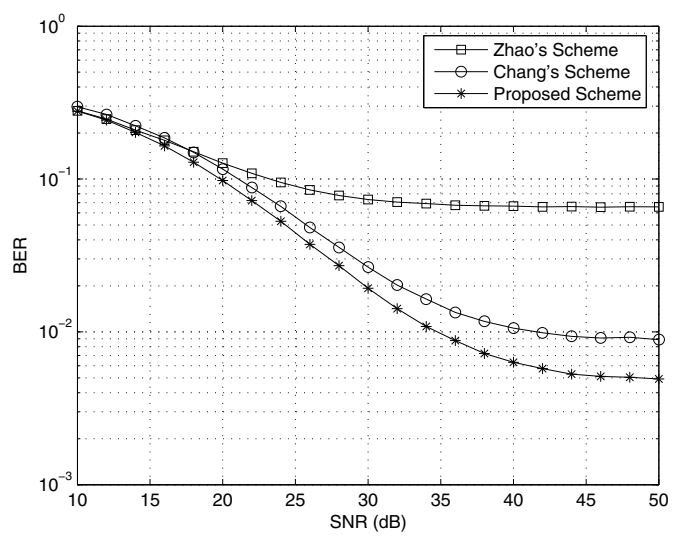

(a) $K=\frac{N}{2}$ and $F_{d}=0.3$

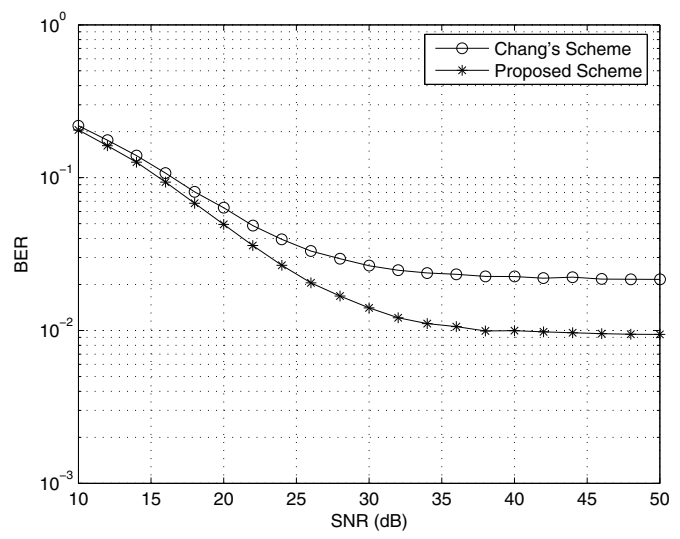

(b) $K=\frac{3}{4} N$ and $F_{d}=0.06$

Fig. 4. BER versus SNR

fractional RRFs, we plot the minimum SIR over the equivalent subchannels in Fig. 3(b) since it restricts the overall system performance. Figure 3(b) indicates that for fractional RRFs, the performance of the Chang's scheme degrades significantly in low $F_{d}$ regions. As a result, the SIR gain of the proposed reduced-rate transmission over the Chang's scheme increases considerably as $F_{d}$ decreases. Moreover, it is observed that the $\frac{3}{4}$-rate scheme has a much lower SIR than the half-rate one. As explained in Section IV-B, time-domain variation of the wireless channel cannot be well eliminated by a fractional RRF. Therefore, the $\frac{3}{4}$-rate scheme increases the transmission rate at the expense of degraded ICI mitigation capability.

Figure 4(a) shows the BER versus SNR when $K=\frac{N}{2}$ and $F_{d}=0.30$; Figure 4(b) shows the BER versus SNR when $K=\frac{3}{4} N$ and $F_{d}=0.06$. For both half-rate and $\frac{3}{4}$-rate schemes, 4 equally spaced equivalent subchannels are loaded with pilots for channel estimation. Furthermore, the 64-QAM and the 16-QAM modulation means are applied in the half-rate and the $\frac{3}{4}$-rate schemes, respectively. At the receiver, the zeroforcing detection is applied and no error-correcting coding is implemented. From Fig. 4, we observe that the proposed reduced-rate transmission effectively lowers the BER floor for both the half-rate and the $\frac{3}{4}$-rate schemes. Moreover, an SNR gain of around $2 \mathrm{~dB}$ over the Chang's scheme is observed even before the BER floor occurs.

\section{Conclusions}

In this paper, we have developed a general reduced-rate OFDM transmission scheme for ICI mitigation in a highmobility environment. By transmit and receive preprocessing, we have transformed the original $N$-subcarrier OFDM system into an equivalent $K$-subcarrier one with significantly reduced ICI. In particular, we have developed a general structure of transmit and receive preprocessing matrices to ensure that all equivalent subchannels share a common average SIR, which is further maximized based on the channel statistics. Both numerical and simulation results have demonstrated the significant performance improvement of the proposed reduced-rate transmission over the existing ICI self-cancellation schemes.

\section{REFERENCES}

[1] M. Russell and G. L. Stüber, "Interchannel interference analysis of OFDM in a mobile environment," in Proc. IEEE Veh. Tech. Conf., pp. 820-824, July 1995.

[2] G. Y. Li and L. Cimini, "Bounds on the inter-channel interference of OFDM in time-varying impairments," IEEE Trans. Wireless Commun., vol. 49, pp. 401-404, Mar. 2001.

[3] W. Jeon, K. Chang, and Y. Cho, "An equalization technique for orthogonal frequency-division multiplexing systems in time-variant multipath channels," IEEE Trans. Commun., vol. 47, pp. 27-32, Jan. 1999.

[4] Y. Mostofi and D. C. Cox, "ICI mitigation for pilot-aided OFDM mobile systems," IEEE Trans. Wireless Commun., vol. 4, pp. 765-774, Mar. 2005.

[5] A. Stamoulis, S. Diggavi, and N. Al-Dhahir, "Intercarrier interference in MIMO OFDM," IEEE Trans. Signal Processing, vol. 50, pp. 2451-2464, Oct. 2002.

[6] Y. Zhao and S. G. Haggman, "Intercarrier interference compression in OFDM communication systems by using correlative coding," IEEE Commun. Let., vol. 2, pp. 214-216, Aug. 1998.

[7] H. Zhang and G. Y. Li, "Optimum frequency-domain partial response encoding in OFDM system," IEEE Trans. Commun., vol. 51, pp. 1064 1068, July 2003.

[8] Y. Zhao and S. G. Haggman, "Intercarrier interference self-cancellation scheme for OFDM mobile communication systems," IEEE Trans. Commun., vol. 49, pp. 1185-1191, July 2001.

[9] M. X. Chang, "A novel algorithm of inter-subchannel interference selfcancellation for OFDM systems," IEEE Trans. Wireless Commun., vol. 6 , pp. 2881-2893, Aug. 2007.

[10] J. Ma, G. Y. Li, P. Orlik, and J. Y. Zhang, "Reduced-rate OFDM transmission for ICI self-cancellation over high-mobility fading channels," submitted to IEEE Trans. Wireless Commun., Dec. 2010. 